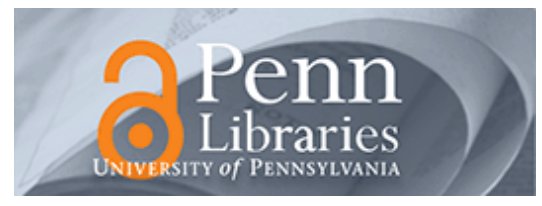

University of Pennsylvania

ScholarlyCommons

10-11-2006

\title{
Colloidal Electroconvection in a Thin Horizontal Cell. II. Bulk Electroconvection of Water During Parallel-Plate Electrolysis
}

\author{
Yilong Han \\ University of Pennsylvania \\ David G. Grier \\ New York University
}

Follow this and additional works at: https://repository.upenn.edu/physics_papers

Part of the Physics Commons

\section{Recommended Citation}

Han, Y., \& Grier, D. G. (2006). Colloidal Electroconvection in a Thin Horizontal Cell. II. Bulk

Electroconvection of Water During Parallel-Plate Electrolysis. Retrieved from https://repository.upenn.edu/ physics_papers/183

Suggested Citation:

Han, Y. and Grier, D.G. (2006). Colloidal electroconvection in a thin horizontal cell. II. Bulk electroconvection of water during parallel-plate electrolysis. The Journal of Chemical Physics 125, 144707.

(C) 2006 American Institute of Physics. This article may be downloaded for personal use only. Any other use requires prior permission of the author and the American Institute of Physics. The following article appeared in The Journal of Chemical Physics and may be found at http://dx.doi.org/10.1063/1.2349486.

This paper is posted at ScholarlyCommons. https://repository.upenn.edu/physics_papers/183

For more information, please contact repository@pobox.upenn.edu. 


\title{
Colloidal Electroconvection in a Thin Horizontal Cell. II. Bulk Electroconvection of Water During Parallel-Plate Electrolysis
}

\author{
Abstract \\ We recently have reported [J. Chem. Phys. 122, 164701 (2005)] a family of electroconvective patterns that \\ arise when charge-stabilized colloidal dispersions are driven by constant (dc) vertical electric fields. \\ Competition between gravity and electrokinetic forces acting on the individual spheres in this system \\ leads to the formation of highly organized convective instabilities involving hundreds of spheres. Here, we \\ report a distinct class of electroconvective patterns that emerge in confined aqueous dispersions at \\ higher biases. These qualitatively resemble the honeycomb and labyrinthine patterns formed during \\ thermally driven Rayleigh-Bénard convection, but arise from a distinct mechanism. Unlike the localized \\ colloidal electroconvective patterns observed at lower biases, moreover, these system-spanning patterns \\ form even without dispersed colloidal particles. Rather, they appear to result from an underlying \\ electroconvective instability during electrolysis in the parallel plate geometry. This contrasts with recent \\ theoretical results suggesting that simple electrolytes are linearly stable against electroconvection.

\section{Disciplines} \\ Physical Sciences and Mathematics | Physics

\section{Comments} \\ Suggested Citation: \\ Han, Y. and Grier, D.G. (2006). Colloidal electroconvection in a thin horizontal cell. II. Bulk \\ electroconvection of water during parallel-plate electrolysis. The Journal of Chemical Physics 125, \\ 144707. \\ (C) 2006 American Institute of Physics. This article may be downloaded for personal use only. Any other \\ use requires prior permission of the author and the American Institute of Physics. The following article \\ appeared in The Journal of Chemical Physics and may be found at http://dx.doi.org/10.1063/1.2349486.
}




\title{
Colloidal electroconvection in a thin horizontal cell. II. Bulk electroconvection of water during parallel-plate electrolysis
}

\author{
Yilong Han \\ Department of Physics and Astronomy, University of Pennsylvania, Philadelphia, Pennsylvania 19104 \\ David G. Grier ${ }^{a)}$ \\ Department of Physics, New York University, New York, New York 10003 \\ and Center for Soft Matter Research, New York University, New York, New York 10003
}

(Received 16 May 2006; accepted 11 August 2006; published online 11 October 2006)

\begin{abstract}
We recently have reported [J. Chem. Phys. 122, 164701 (2005)] a family of electroconvective patterns that arise when charge-stabilized colloidal dispersions are driven by constant (dc) vertical electric fields. Competition between gravity and electrokinetic forces acting on the individual spheres in this system leads to the formation of highly organized convective instabilities involving hundreds of spheres. Here, we report a distinct class of electroconvective patterns that emerge in confined aqueous dispersions at higher biases. These qualitatively resemble the honeycomb and labyrinthine patterns formed during thermally driven Rayleigh-Bénard convection, but arise from a distinct mechanism. Unlike the localized colloidal electroconvective patterns observed at lower biases, moreover, these system-spanning patterns form even without dispersed colloidal particles. Rather, they appear to result from an underlying electroconvective instability during electrolysis in the parallel plate geometry. This contrasts with recent theoretical results suggesting that simple electrolytes are linearly stable against electroconvection. (C) 2006 American Institute of Physics. [DOI: $10.1063 / 1.2349486$ ]
\end{abstract}

\section{INTRODUCTION}

Several processes occur when parallel plate electrodes are used to apply uniform electric fields to aqueous electrolytes. Below the threshold for electrochemical reactions, dissolved ions redistribute around the electrodes and screen the field in the bulk. At higher biases, water dissociates into hydroxyl and hydronium ions that flow to the oppositely charged electrodes, where they recombine into molecular gases that dissolve into the electrolyte. The anionic and cationic fluxes ordinarily intermingle to maintain local electroneutrality. This not only minimizes the system's electrostatic energy but also helps to maintain mechanical stability because the counterpropagating fluxes exert no net force on the water.

The mechanical stability of steady hydrolysis can be disrupted by adding macroscopic charged objects such as dispersed colloidal spheres. The spheres do not engage in electrochemical reactions, and their fixed charges favor a departure from local electroneutrality in the surrounding electrolyte. ${ }^{1}$ Once the ionic fluxes are spatially separated, they entrain counterpropagating flows of water around the spheres. ${ }^{1-3}$ These flows exert hydrodynamic forces on the spheres, and their motions, in turn, redirect the flows. ${ }^{2-5} \mathrm{We}$ previously reported ${ }^{6,7}$ that the interplay of microscopic electrohydrodynamic flows around individual spheres can give rise to large-scale convective instabilities characterized by highly organized flow patterns involving thousands of spheres. These colloidal electroconvective patterns form at

${ }^{a)}$ Electronic mail: david.grier@nyu. edu biases just above the threshold for steady electrolysis, and their structure depends on the properties and concentration of the colloidal spheres. Consequently, they appear not to reflect underlying electroconvective instabilities in the electrolyte itself. 6,7

The present article describes a distinct category of bulk electroconvective patterns that form at higher biases even without dispersed colloidal spheres. Their appearance demonstrates that bulk electroconvective instabilities can occur in simple electrolytes under steady driving by uniform fields. These observations therefore provide an experimental resolution to a long-standing debate regarding the mechanical stability of simple electrolytes in the parallel-plate geometry. ${ }^{8-10}$

Qualitatively similar instabilities have been observed during the electrodeposition of copper from aqueous electrolytes in the parallel plate geometry. ${ }^{11}$ In that case, convection was attributed to density gradients resulting from currentdriven concentration gradients in the dissolved copper ions. Electrohydrodynamically driven convection also has been reported during the electrolysis of model nonaqueous electrolytes ${ }^{12,13}$ in vertical slit pores. In this case, fluid flow appears to be nucleated by a charge injection mechanism, and does not involve gravity.

Until recently, the onset of electroconvection without gravity in simple electrolytes was believed to resemble the intrinsic convective instability in plasmas. ${ }^{8}$ Unlike plasma convection, however, the electrohydrodynamic instability in electrolytes is entirely suppressed by homogeneous boundary conditions. ${ }^{9}$ Bulk gravity-free electroconvection in simple electrolytes therefore should occur only in the presence of 


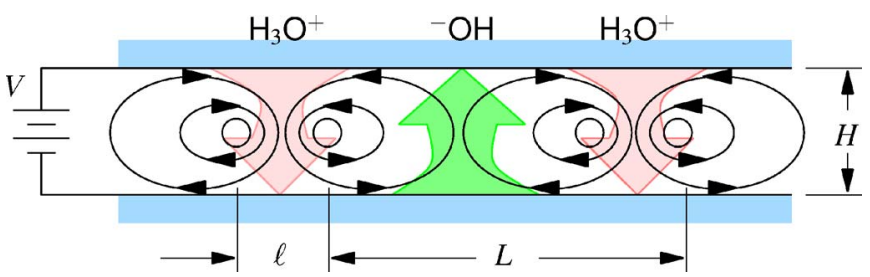

FIG. 1. (Color online) Schematic cross section of the system showing counterrotating convection rolls between parallel transparent conducting surfaces, driven by counterpropagating ionic fluxes. Negatively charged spheres concentrate near the flux of hydrolytically generated hydronium ions.

inhomogeneous boundary conditions, ${ }^{10}$ and the resulting patterns should be pinned to the underlying inhomogeneities. This is consistent with observation of bulk electroconvection during the electrodeposition of branched metallic structures from aqueous electrolytes. ${ }^{14,15}$ We find, by contrast, that electrolysis of pure water in a horizontal planar slit pore yields drifting cellular electroconvection patterns not obviously associated with electrode structures under conditions not conducive to plasmalike convective instabilities. ${ }^{8,9}$

Because the high-bias electrohydrodynamic patterns described below emerge from and supplant the low-bias colloidal electroconvective instabilities we have previously described, ${ }^{6,7}$ we organize our present observations as extensions of that work. The patterns' phenomenology then allows us to distinguish the pattern forming mechanism. Section 2 describes the experimental apparatus, whose ability to create large-scale electroconvective patterns is discussed in Sec. III. Observations described in this section also demonstrate that the electroconvective patterns are largely insensitive to the dispersed colloids' properties or concentration. Section IV discusses the patterns' dependence on other experimental conditions such as $p \mathrm{H}$ and electrode uniformity. The full set of observations is summarized in Sec. $\mathrm{V}$ to provide an overview of electroconvective pattern formation in water.

\section{EXPERIMENTAL SYSTEM}

Our experimental system was described in Ref. 7 and is shown schematically in Fig. 1. Each sample consists of aqueous dispersions of micrometer-sized colloidal spheres confined to a thin horizontal layer between a glass slide and a glass cover slip. Both inner surfaces are coated with 10-nm-thick gold electrodes on 10-nm-thick titanium wetting layers. These electrodes are transparent enough that individual particles can be imaged with a standard light microscope and their motions recorded for analysis. Their $50 \Omega / \square$ resistivity is small enough to yield an essentially uniform electric field across the $H=\mathcal{O}(100) \mu \mathrm{m}$ interelectrode gap in the roughly $4 \mathrm{~cm}^{2}$ accessible sample area.

Sealed electrochemical cells were mounted on the stage of a Zeiss S100TV Axiovert inverted optical microscope. We applied constant (dc) voltages across the electrodes and recorded the resulting time evolution of the distribution of spheres in the gap. The resulting electric field is vertical and oriented with gravity to within $0.1^{\circ}$.

Water dissociates in these sample cells at biases above $2.5 \pm 0.2 \mathrm{~V}$, with bubbles forming at the electrodes after a

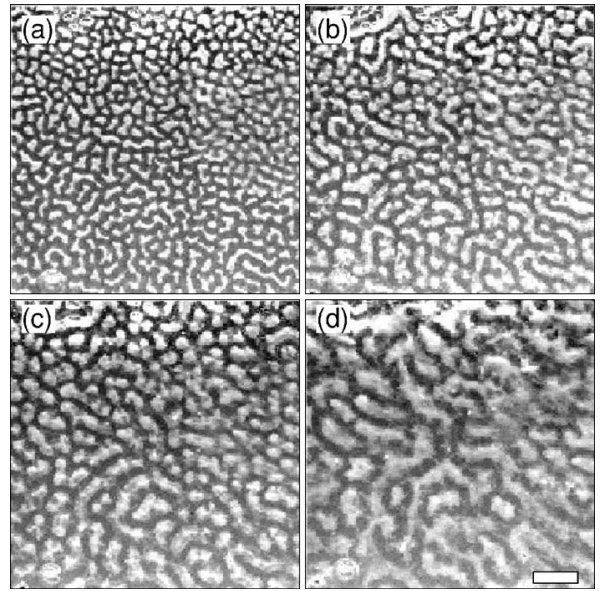

FIG. 2. Labyrinthine patterns of $3.0 \mu \mathrm{m}$ diameter silica spheres at $\phi$ $\approx 120 \%$ in a cell $H=200 \mu \mathrm{m}$ thick. (a) $3.0 \mathrm{~V}$ : a microscopic labyrinth consisting of microscopic clusters. (b) $5.0 \mathrm{~V}$ : a macroscopic labyrinth composed of counterrotating double rolls. (c) $7.0 \mathrm{~V}$. (d) $9.0 \mathrm{~V}$. Lighter regions contain a higher concentration of spheres. Scale bar indicates $1 \mathrm{~mm}$.

few minutes. Because bubbles disrupt the electrohydrodynamically driven patterns, the results reported below were obtained in the interval before macroscopic bubbles evolve. Adopting the conventions from Ref. 7, we report the number density $\phi$ of sedimented colloidal silica spheres in units of the areal density of a close-packed layer, with $\phi=200 \%$ referring to two complete layers. We define the applied dc voltage to be positive if the upper electrode is positive.

\section{BULK CONVECTION: DOUBLE-ROLL LABYRINTHS, HONEYCOMBS, AND MORE}

System-spanning convection rolls appear at biases above roughly $4 \mathrm{~V}$ and disrupt any self-regulating microscopic clusters formed at lower voltages. ${ }^{6,7}$ The rolls organize the particles into macroscopic patterns with features ranging in size from 0.2 to $2 \mathrm{~mm}$. Positive biases in the range of 4-10 V produce bulk patterns such as labyrinths and cellular honeycomb rolls. Unlike microscopic patterns, which form only for charged spheres, and even then only for a limited range of diameters and densities and concentrations, the same large-scale convective patterns form for colloidal spheres with various compositions and characteristics. Patterns similar to those described below form in aqueous dispersions of negatively charged silica spheres, sulfateterminated polystyrene sulfate (PS) spheres, carboxylated PS spheres, weakly charged polymethylmethacrylate (PMMA) spheres, and neutral hydroxylterminated PS spheres. Whereas microscopic patterns arise as cooperative manysphere phenomena over a very limited range of concentrations $\phi$, macroscopic patterns can be discerned in the motions of individual spheres at vanishing low number density, as well as at number densities exceeding $\phi=300 \%$. Consequently, dispersed colloidal spheres appear to contribute little to the pattern formation mechanism in the high-bias regime, and the macroscopic patterns we observe instead appear to result from convective instabilities in the electrolyte itself. Surprisingly, these instabilities appear not to have been described previously. 


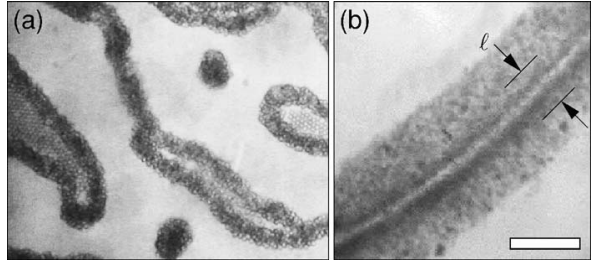

FIG. 3. Microscopic views of labyrinthine colloidal convection rolls formed by $3.0 \mu \mathrm{m}$ diameter silica spheres at $\phi \approx 120 \%$ in a layer of water $H$ $=200 \mu \mathrm{m}$ thick. (a) Uncorrelated colloidal vortices at $V=2.6 \mathrm{~V}$. (b) A double-roll convection roll at $V=4.0 \mu \mathrm{m}$. The arrows indicate the inter-roll separation, as in Fig. 1. The scale bar indicates $50 \mu \mathrm{m}$.

Figures 2(a)-2(d) show the evolution of microscopic colloidal vortices into macroscopic labyrinthine electroconvection rolls with increasing voltage for a sample of $3 \mu \mathrm{m}$ diameter silica spheres at an initial number density of $\phi$ $=120 \%$ in a parallel-electrode cell at $H=200 \mu \mathrm{m}$. The labyrinths' spatial period increases with the bias, as can be seen in Figs. 2(b)-2(d).

Although the labyrinth in Fig. 2(b) at $5 \mathrm{~V}$ qualitatively resembles the lower-bias pattern at $3 \mathrm{~V}$ in Fig. 2(a), their microscopic structure is quite different. The bright features in Fig. 2(a) consist of individual wormlike colloidal vortices, whose microstructure can be seen in Fig. 3(a). These structures were described in Ref. 7 and consist of rolling clusters of spheres hovering near the middle of the sample volume. Individual spheres appear dark in these images, so that the bright domains in Fig. 2 appear dark here. Although the circulation direction is well defined in individual wormlike colloidal vortices, neighboring vortices' circulation direction is uncorrelated. By contrast, the bright domains in Figs. 2(b)-2(d) consist of paired counterrotating convection rolls, an example of which appears in Fig. 3(b). Unlike wormlike colloidal vortices, these rolls span the sample thickness $H$ and are correlated throughout the observable sample area. Charged colloidal particles, such as the silica spheres in Figs. 2 and 3, are concentrated at the interface between the counterrotating rolls and flow downward along the interface at positive bias, as shown schematically in Fig. 1.

Double-roll labyrinths replace wormlike colloidal vortices at roughly $4 \mathrm{~V}$. Convection becomes more vigorous as the bias increases, and rolls tend to evolve into itinerant tornadolike structures, such as the example in Fig. 4 by roughly $6 \mathrm{~V}$. These structures twirl rapidly, completing more than $1 \mathrm{cycle} / \mathrm{s}$. They also can travel through the sample at several $\mathrm{mm} / \mathrm{s}$ as bubbles nucleate and expand at the electrodes.

Whereas microscopic colloidal vortices only form under positive bias, macroscopic electroconvection can be driven by biases of either sign. Suddenly inverting the bias on an already-formed electroconvection pattern reverses the direction of circulation in about $1 \mathrm{~s}$ without disrupting the largescale pattern. Negative biases also tend to push spheres onto the lower electrode, where they eventually deposit. This limits opportunities to study electroconvection at negative bias in our system.

Figure 5 shows how a microscopic colloidal vortex ring is transformed into a pair of convection rolls as the bias increases. The colloidal vortex ring in Fig. 5(a) formed spon-

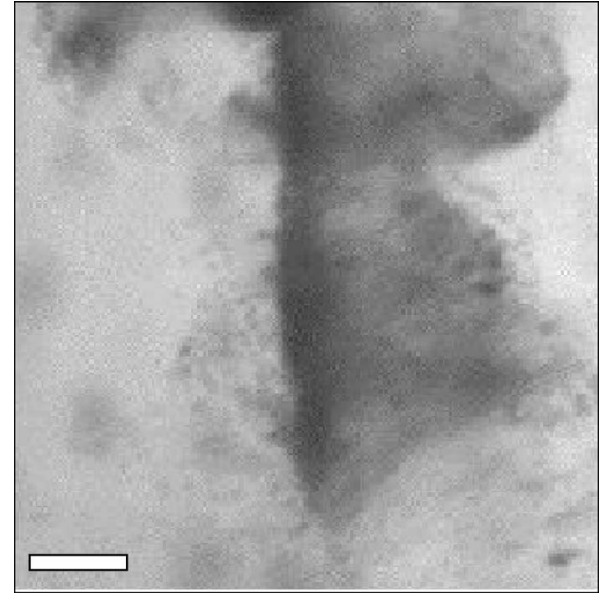

FIG. 4. Electroconvective tornado formed at $6 \mathrm{~V}$ in a cell with $H$ $=200 \mu \mathrm{m}$. Scale bar indicates $50 \mu \mathrm{m}$.

taneously from a dispersion of $2.9 \mu \mathrm{m}$ diameter silica spheres in a cell with $H=200 \mu \mathrm{m}$ under $3.6 \mathrm{~V}$ bias. This $80 \mu \mathrm{m}$ diameter cluster consists of a circulating toroidal vortex of spheres. This vortex ring does not span the sample's thickness, but rather is stably levitated to about $40 \mu \mathrm{m}$ above the lower electrode. Spheres rise near the cluster's center and fall back along the outer edge, completing 1 cycle in roughly $2 \mathrm{~s}$. Increasing the bias continuously to $4.8 \mathrm{~V}$ over several seconds speeds the circulation until finally the toroid elongates and breaks into two counterrotating rolls that span the $H=200 \mu \mathrm{m}$ sample thickness, as shown in Fig. 5(b).

\section{A. Dependence on sphere diameter}

Under the same conditions that $3 \mu \mathrm{m}$ diameter silica spheres form well-organized microscopic colloidal vortices, $1.58 \mu \mathrm{m}$ diameter silica spheres form only tumbling clouds. ${ }^{6,7}$ Although these clouds show none of the internal organization of colloidal vortices, they nevertheless tend to organize themselves into labyrinthine patterns on substantially larger length scales, as can be seen in Fig. 6(a). Like the labyrinths formed by larger spheres, these patterns also coarsen with increasing bias. By about $10 \mathrm{~V}$, however, the smaller lighter particles are driven onto the upper electrode, where they stick irreversibly. These adsorbed spheres typi-

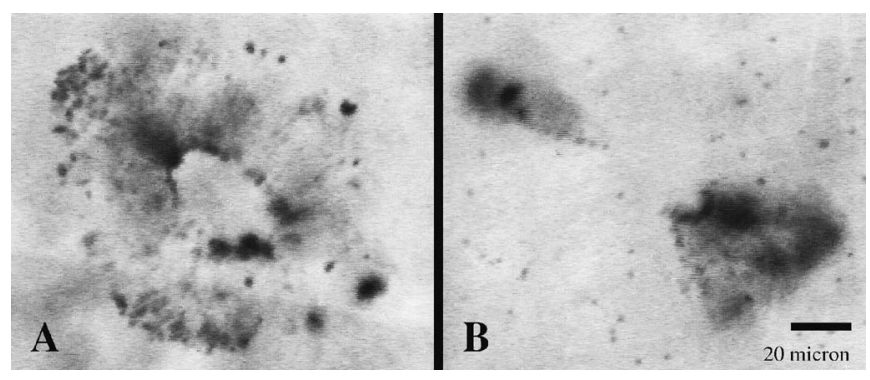

FIG. 5. Transforming a colloidal vortex ring of $2.9 \mu \mathrm{m}$ diameter silica spheres into paired convection rolls with increasing bias. (a) $3.6 \mathrm{~V}$ : colloidal vortex ring. (b) $4.8 \mathrm{~V}$ : the corona breaks into rapidly circulating double rolls. 


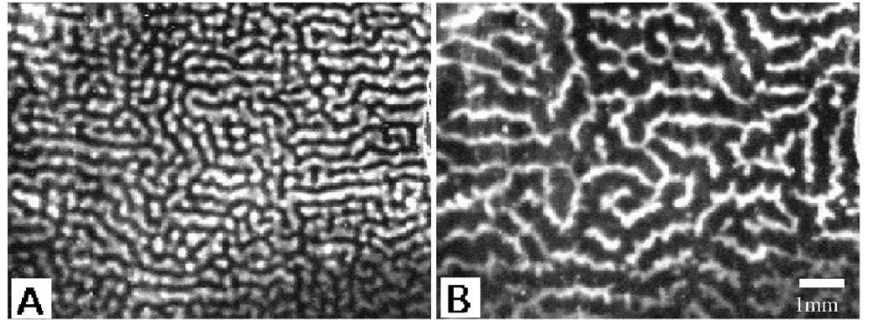

FIG. 6. Labyrinthine patterns of $1.58 \mu \mathrm{m}$ diameter silica spheres. $H$ $=200 \mu \mathrm{m}$. $\phi \approx 50 \%$. (a) $4.0 \mathrm{~V}$ : labyrinthine convection rolls in the bulk (b) $10 \mathrm{~V}$ : labyrinth of crystallites deposited on the upper electrode.

cally are arranged into static labyrinthine domains with crystalline microstructure, an example of which appears in Fig. $6(b)$.

\section{B. Dependence on sphere composition}

To avoid particle deposition under negative bias conditions, we repeated these experiments with $0.5 \mu \mathrm{m}$ diameter PS spheres, whose density of $1.05 \mathrm{~g} / \mathrm{cm}^{3}$ is more closely matched to that of water. These particles form labyrinthine patterns comparable to those in Fig. 2 under comparable positive biases. Figure 7(a) shows that these particles also form steady-state labyrinths in the bulk at $-6.0 \mathrm{~V}$. A similar trend of domain coarsening with increasing driving also is observed with negative biases. These observations suggest that gravity plays no significant role in establishing the observed electrohydrodynamic convection patterns. They also confirm that the patterns are sensitive to neither the spheres' density nor their diameter.

Labyrinth coarsening appears not to be reversible. Reducing the bias to $-4.0 \mathrm{~V}$ might be expected to select a finergrained pattern. With the coarser pattern already established, however, the result is to coalesce the colloid-rich convection rolls into thinner domains, separated by wider margins, as shown in Fig. 7(b).

\section{Concentration dependence: Honeycomb patterns}

Although the nature of the electroconvective patterns formed at a given bias does not depend on the spheres' diameter, it does depend somewhat on their concentration, as can be seen in Fig. 8. Honeycomb patterns, such as the example in Fig. 8(a), appear more often at higher sphere concentrations. These periodic cellular patterns are characterized by three-way junctions between double convection rolls. In other respects, they resemble the labyrinthine patterns. In

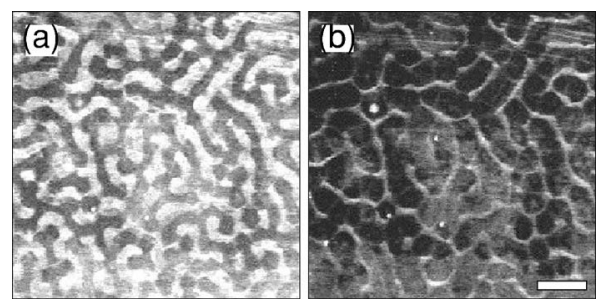

FIG. 7. Labyrinthine electrohydrodynamic convection of $0.5 \mu \mathrm{m}$ diameter PS spheres in a sample with $H=390 \mu \mathrm{m}$. (a) $-6.0 \mathrm{~V}$ : labyrinth in the bulk (b) Effect of reducing the bias to $-4.0 \mathrm{~V}$. Scale bar indicates $1 \mathrm{~mm}$.
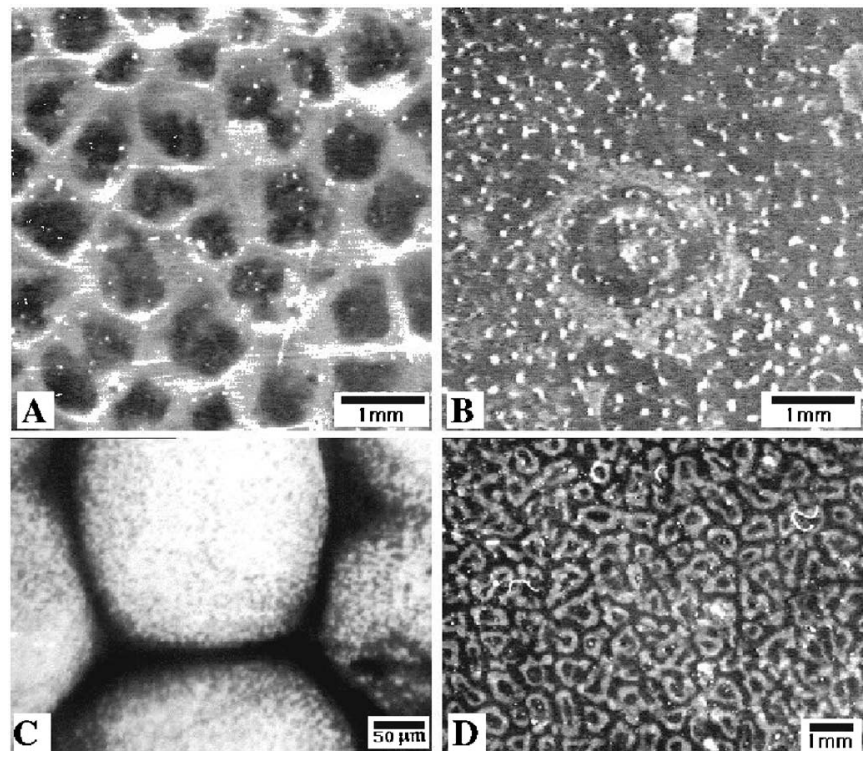

FIG. 8. Honeycomb and dot patterns. Dense regions of spheres appear white in (a)-(d), but dark in (c). (a) $1.8 \mu \mathrm{m}$ PS sphere, $H=390 \mu \mathrm{m}$, and $4.0 \mathrm{~V}$. Typical honeycomb pattern formed at high number density. (b) $1.8 \mu \mathrm{m}$ PS spheres, $H=90 \mu \mathrm{m}, 8.0 \mathrm{~V}$, and $\phi \approx 10 \%$. Spot patterns (c) $1.8 \mu \mathrm{m}$ PS spheres at the upper electrode for $H=390 \mu \mathrm{m}$ and $3.0 \mathrm{~V}$. Multilayer crystals are apparent in dark areas. (d) $0.29 \mu \mathrm{m}$ silica spheres, $H=390 \mu \mathrm{m}$, and $10 \mathrm{~V}$. Convective cloudy rings.

particular, the dispersed spheres continue to be concentrated near the region of fastest circulation at the junction between adjoining rolls.

Lower concentrations favor discontinuous honeycomb patterns, which appear in Fig. 8(b), with spheres concentrated at vertices joining three convection rolls rather than at interfaces between two. Similar polka dot patterns also are obtained with 0.29 or $7.0 \mu \mathrm{m}$ diameter PS spheres in a $390 \mu \mathrm{m}$ thick cell at comparable filling fractions $\phi$.

As for the labyrinthine patterns, bulk honeycomb patterns gradually deposit their spheres onto the electrodes. The image in Fig. 8(c) shows $1.8 \mu \mathrm{m}$ PS spheres at the upper electrode of a $H=390 \mu \mathrm{m}$ thick sample during bulk honeycomb convection at 3.0 V. Dark regions in this image consist of multilayer colloidal crystals. Removing the bias frees all but the uppermost layer to sediment back to the lower electrode. The remaining crystalline layer is irreversibly bound to the upper electrode and retains the convection rolls' honeycomb structure.

Honeycomb rolls form just as readily with sulfate terminated PS, carboxylated PS, neutral hydroxyl-terminated PS, and PMMA particles. In all cases, applying a positive bias causes particles to flow upward within each cell's core and to circulate back downward along the borders between neighboring cells.

\section{Dependence on sample geometry}

Figures 9 and 10 show that bulk labyrinthine patterns are not strongly affected by the boundaries of the observation channel, but rather extend right up to the walls. Nor are labyrinths oriented in any obvious way by the boundaries. The images in Figs. 9 and 10 were obtained in two cells of $1 \mathrm{~mm}$ deep, the first with in-plane dimensions of 4 


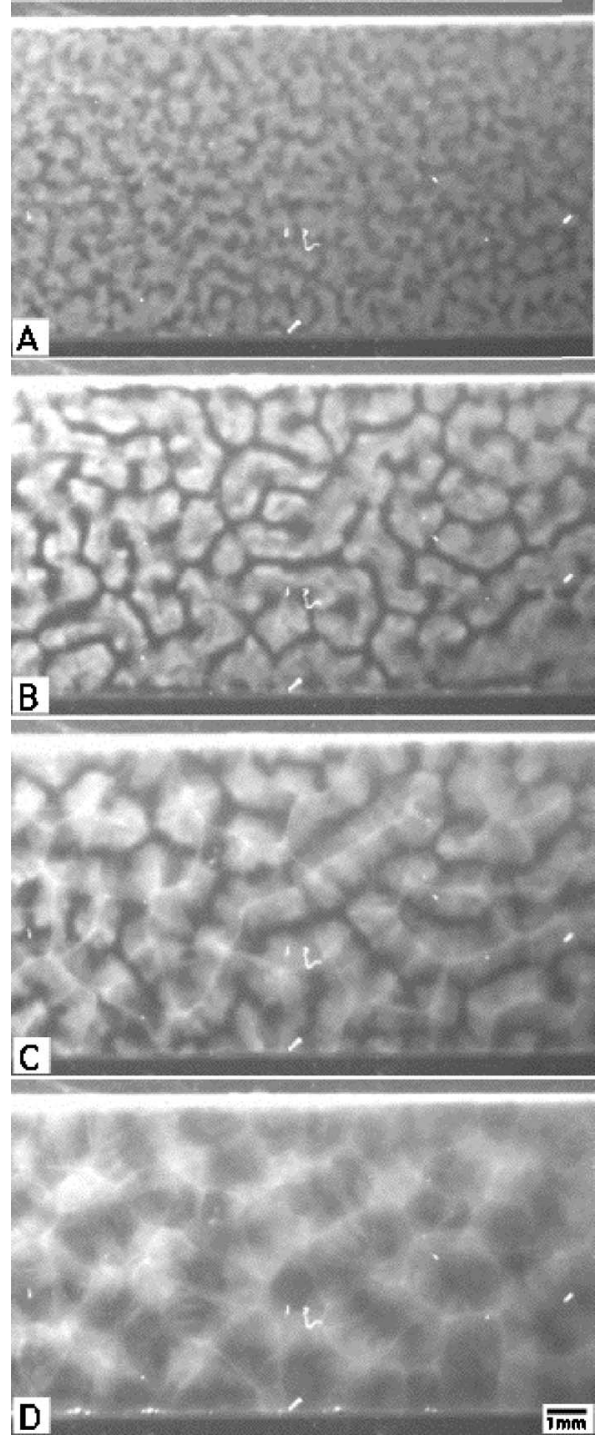

FIG. 9. Labyrinth in the $H=1 \mathrm{~mm}$ cell (high particle density, wide cell). $1.5 \mu \mathrm{m}$ diameter silica spheres. White areas have higher particle density. (a) $4.0 \mathrm{~V}$, (b) $8.0 \mathrm{~V}$, (c) $12.0 \mathrm{~V}$, and (d) $20.0 \mathrm{~V}$.

$\times 0.9 \mathrm{~cm}^{2}$ and the second of $4 \times 0.3 \mathrm{~cm}^{2}$. Despite this difference, both cells produced similar patterns, with any differences being ascribable to differences in particles density. This further illustrates that the transverse boundary condition has little influence on bulk pattern formation in the electroconvective regime. Figures 9 and 10 also highlight the wide range of parameters under which labyrinths form, including cells at least as deep as $H=1 \mathrm{~mm}$ and at biases as high as $20 \mathrm{~V}$.

\section{THE ROLE OF CHARGE, IONIC CONCENTRATION, AND $p H$}

Trends in electroconvective pattern formation suggest some minor role for the dispersed colloidal particles in initiating the instability. The particles' charge, however, appears to be surprisingly unimportant. Bulk honeycomb patterns form just as easily with electrically neutral hydroxylterminated polystyrene particles as with highly charged sulfate-terminated particles. This contrasts with the forma-

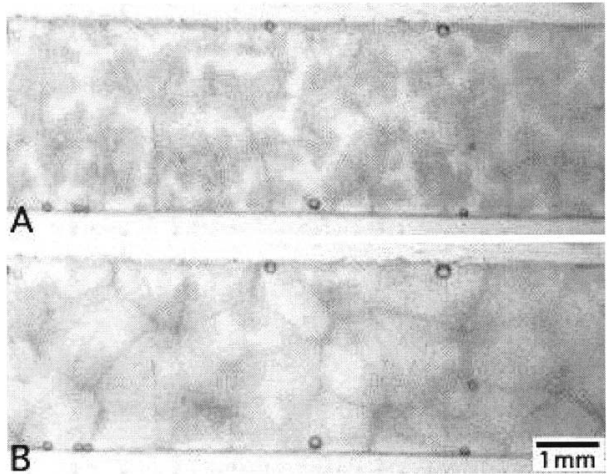

FIG. 10. Labyrinth in the $H=1 \mathrm{~mm}$ cell (low particle density, narrow cell). $1.5 \mu \mathrm{m}$ diameter silica spheres. Dark areas have higher particle density. (a) $8.0 \mathrm{~V}$ and (b) $16.0 \mathrm{~V}$.

tion of microscopic levitated clusters in our previous reports, ${ }^{6,7}$ to which the particles' charge proved integral. It suggests that bulk honeycomb or labyrinthine convection patterns are due to an instability in the electrolyte itself and that the particles serve only as visible tracers of these flows.

Dispersed particles also may serve as tracers for other properties as well. For instance, charged spheres are more likely to congregate in regions of high counterion concentration. In the absence of added salt, $\mathrm{H}_{3} \mathrm{O}^{+}$and $\mathrm{OH}^{-}$are the predominant ionic species in our systems, so that the spheres thus may serve as $p \mathrm{H}$ tracers. To test this idea, we performed experiments on dispersions of highly charged polystyrene sulfate spheres with molecular $p \mathrm{H}$ indicators and in cells with indium tin oxide (ITO) electrodes.

Adding trace amount of the $p \mathrm{H}$ indicator methyl red into the cell does not affect the sequence of macroscopic patterns formed with increasing bias. However, the milky white bulk labyrinth that forms in an unlabeled suspension appears bright red with added indicator. The regions of low sphere concentration, on the other hand, appear yellow. Methyl red turns yellow at $p \mathrm{H}>6.0$, red at $p \mathrm{H}<4.4$, and orange in between. Thus the $p \mathrm{H}$ in the sphere-rich areas is lower than 4.4, and that between the dense stripes exceeds 6.0.

The highly negatively charged spheres themselves act as strong acids. The color gradients therefore might reflect the influence of the spheres on the $p \mathrm{H}$, rather than the ionic concentrations' influence on the spheres' distribution. Repeating the experiment with more weakly charged silica spheres yields similar profiles in the distribution of spheres, but with much weaker color gradients. Repeating the experiment with no spheres at all yields no visible patterns in the indicator even though related experiments with very small numbers of dispersed spheres still reveal patterns of convection rolls under comparable conditions. The absence of visible color gradients under these conditions does not guarantee the absence of $p \mathrm{H}$ gradients. Rather, it may reflect the difficulty of detecting subtle color changes in thin electroconvection cells without the additional contrast provided by concentrations of spheres.

Consequently, we performed additional experiments using electrodes fabricated from thin films of ITO. ITO is a transparent conductor that develops a tint under oxidizing conditions that ranges from light yellow to dark gray depend- 

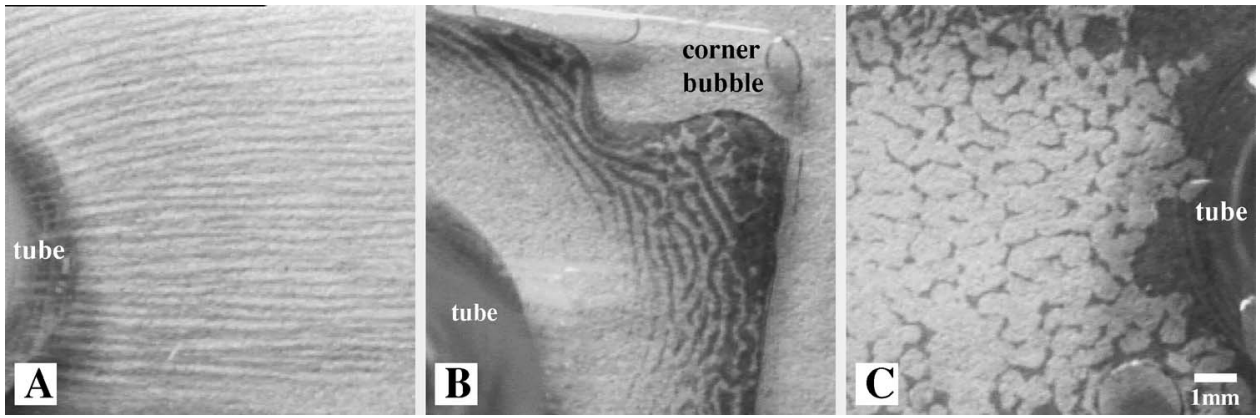

FIG. 11. Oxidation patterns on the lower, negatively biased ITO electrode. Darker areas have been oxidized and lighter, transparent areas remain in the initial unoxidized state. (a) The pattern in the central region of the electrode. (b) A region near a corner of the sample cell. (c) Central region of another electrode after $30 \mathrm{sec}$ at $8 \mathrm{~V}$ bias.

ing on the degree of oxidation. Colloidal dispersions form labyrinthine and honeycomb convection patterns just as readily with the ITO electrodes as with the gold. In this case, however, the ITO electrodes show clear signs of oxidation, with yellow bands replicating the millimeter-scale patterns displayed by the convecting spheres. Repeating the experiment in de-ionized water without dispersed spheres yields comparable results, with well defined yellow stripes appearing on the negatively biased lower electrode after just a few seconds of hydrolysis. Typical results appear in Fig. 11.

These observations suggest that water itself undergoes large-scale electroconvective instabilities leading to the formation of labyrinthine and honeycomb patterns. They further suggest that these patterns result from a spatial separation between the anionic and cationic fluxes generated by hydrolysis of water and thus a departure from local electroneutrality.

This large-scale separation of ionic fluxes, depicted schematically in Fig. 1, is consistent with the theory for bulk gravity-free electroconvection of water., ${ }^{9,10}$ The forgoing observations therefore appear to be an experimental realization of this phenomenon. Although dispersed spheres may influence details of the intrinsic electroconvective instability, their presence is not required. This contrasts with the formation of electrohydrodynamically driven colloidal vortices described in Refs. 6 and 7.

If indeed pure water is linearly stable against the onset of electroconvection in the parallel-plate geometry, ${ }^{9}$ then the phenomena described above must be initiated by imperfections in the sample. ${ }^{10}$ Figure 12 , however, shows that convection rolls pass over and across scratches in the thin-film electrodes without distortion. Electroconvection rolls drift as freely across such defects as they do across undamaged regions of the electrode surfaces. Moreover, oxidation patterns in ITO electrodes suggest that electroconvective patterns extend undisturbed all the way to the irregular edges of the sample volume. Irregularities, therefore, may be necessary to bootstrap electroconvection in pure water, ${ }^{10}$ but they do not appear to play a major role in establishing or maintaining the overall pattern once electroconvection is fully developed.

The macroscopic electroconvection patterns discussed here qualitatively resemble patterns generated by thermal gradients during Rayleigh-Bénard convection. ${ }^{16}$ From a mathematical perspective, the analogy is reasonably close. ${ }^{8,9}$ In both cases, diffusive transport establishes a threshold for the convective instability. The principal difference is that heated fluid layers are linearly unstable against Rayleigh-
Bénard convection whereas simple electrolytes are believed to be linearly stable against electroconvection, the stabilization arising from the energetic benefit of maintaining local electroneutrality. ${ }^{9}$

Much of the literature on electroconvection consequently addresses field-induced instabilities in systems where local electroneutrality naturally is violated. In liquid crystal films, for example, charge separation along individual molecules provides the basis for electroconvective instabilities. ${ }^{17}$ Transitions between labyrinthine and cellular patterns in this system are influenced in large part by phase transitions in the liquid crystal itself. ${ }^{17}$ They differ, therefore, from the transitions in electroconvective pattern formation described here, which are not associated with phase transitions in the simple electrolyte.

Another type of electroconvection similar to ours is induced by injecting space charge into apolar liquids. ${ }^{18,19}$ Increasing the bias on such a system yields a sequence of patterns ranging from hexagonal cells to filaments to turbulence. The dielectric liquids used in these experiments have extremely high degree of ionic purity so that space charges will be established during electrical conduction. Both electroneutrality and Ohm's law break down in such systems. By contrast, our aqueous electrolytes are electrically neutral, and their conductivity is high enough that Ohm's law holds. Observing electroconvection in simple electrolytes therefore greatly expands the range of isotropic systems fluids capable of supporting bulk field-driven instabilities.

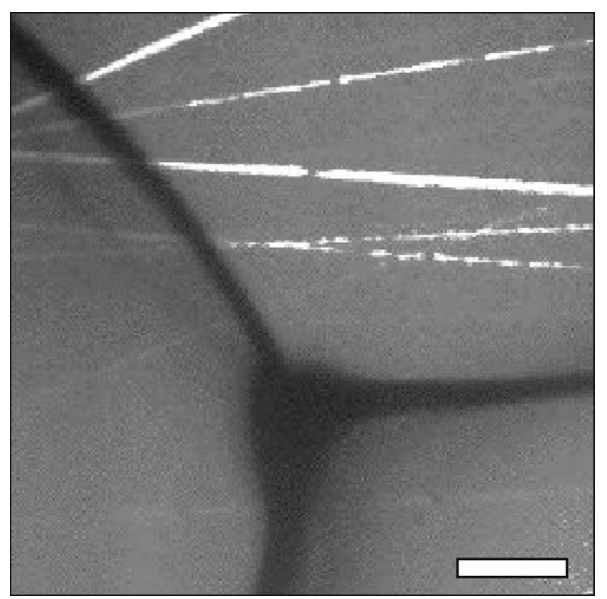

FIG. 12. Magnified view of three dark electroconvective double rolls at the vertex of a honeycomb pattern passing over scratches in the lower electrode. The rolls appear dark in this image, while the scratches appear bright white. Scale bar indicates $50 \mu \mathrm{m}$. 


\section{SUMMARY}

We have observed the emergence of labyrinthine and honeycomb electroconvection patterns with length scales between 0.5 and $2 \mathrm{~mm}$ during the steady-state hydrolysis of water at biases above $4 \mathrm{~V}$ in a parallel plate electrolysis cell. These macroscopic patterns share some features with the microscopic clusters we described in Refs. 6 and 7. Among the similarities are the circulation direction of the convection rolls, the coarsening of the patterns features with increasing bias, and the increase in circulation rate with increasing voltage.

Unlike microscopic colloidal electroconvection, macroscopic electroconvection patterns form under both negative and positive biases. Flipping the sign of the biases on a preformed pattern merely changes the circulation direction. Also unlike microscopic patterns, which form under a very limited range of conditions, electroconvection patterns arise for both charged and uncharged colloids, and for both neutrally buoyant polystyrene spheres and dense silica spheres. On this basis, we conclude that particles are little more than passive tracers for electroconvective flows in this high bias regime.

Experiments with $p \mathrm{H}$ indicator show that regions containing negatively charged colloidal spheres are more acidic. Experiments with ITO electrodes demonstrate that the $p \mathrm{H}$ distribution near electrodes displays the same periodic patterns even without colloid. Consequently, we conclude that these macroscopic patterns constitute an instability of electrolyte itself. Despite the experiment's simplicity and the effect's generality, this process appears not to have been reported previously.

In this paper and Ref. 7, we have demonstrated that a very simple electrokinetic apparatus can form an exceptionally wide range of highly organized dynamic patterns, even under constant uniform driving. The apparent simplicity of colloidal dispersions subjected to constant and uniform fields is deceptive, however, because colloidal electrodynamics during steady hydrolysis involves a great many processes spanning a huge range of length scales.

Consequently, our most surprising result is that robust self-organization emerges from such complicated processes even when the system is driven very far from equilibrium.

\section{ACKNOWLEDGMENT}

This work was supported by the donors of the Petroleum Research Fund of the American Chemical Society.

${ }^{1}$ M. Trau, S. Sankaran, D. A. Saville, and I. A. Aksay, Nature (London) 374, 437 (1995).

${ }^{2}$ M. Trau, D. A. Saville, and I. A. Aksay, Science 272, 706 (1996).

${ }^{3}$ M. Trau, D. A. Saville, and I. A. Aksay, Langmuir 13, 6375 (1997).

${ }^{4}$ M. Böhmer, Langmuir 12, 5747 (1996).

${ }^{5}$ Y. Solomentsev, M. Böhmer, and J. L. Anderson, Langmuir 13, 6058 (1997).

${ }^{6}$ Y. Han and D. G. Grier, Nature (London) 424, 267 (2003).

${ }^{7}$ Y. Han and D. G. Grier, J. Chem. Phys. 122, 164701 (2005).

${ }^{8}$ R. Bruinsma and S. Alexander, J. Chem. Phys. 92, 3074 (1990).

${ }^{9}$ I. Lerman, I. Rubinstein, and B. Zaltzman, Phys. Rev. E 71, 011506 (2005).

${ }^{10}$ T. Pundik, I. Rubinstein, and B. Zaltzman, Phys. Rev. E 72, 061502 (2005).

${ }^{11}$ S. Nakabayashi, M. Yanagida, and K. Uosaki, J. Phys. Chem. 100, 714 (1996).

${ }^{12}$ M. Orlik, J. Rosenmund, K. Doblhofer, and G. Ertl, J. Phys. Chem. B 102, 1397 (1998).

${ }^{13}$ D. Dini, K. Doblhofer, and G. Ertl, Phys. Chem. Chem. Phys. 2, 1183 (2000).

${ }^{14}$ V. Fleury, J.-N. Chazalviel, and M. Rosso, Phys. Rev. Lett. 68, 2492 (1992).

${ }^{15}$ V. Fleury, J.-N. Chazalviel, and M. Rosso, Phys. Rev. E 48, 1279 (1993).

${ }^{16} \mathrm{~S}$. Chandrasekhar, Hydrodynamic and Hydromagnetic Stability (Clarendon, Oxford, 1961).

${ }^{17}$ L. Kramer and W. Pesch, Annu. Rev. Fluid Mech. 27, 515 (1995).

${ }^{18}$ J. C. Lacroix, P. Atten, and E. J. Hopfinger, J. Fluid Mech. 69, 539 (1975).

${ }^{19}$ P. Atten, IEEE Trans. Dielectr. Electr. Insul. 3, 1 (1996). 